\title{
Fractality of Fracture Surfaces
}

\author{
T. Ficker
}

\begin{abstract}
A recently published fractal model of the fracture surfaces of porous materials is discussed, and a series of explanatory remarks are added. The model has revealed a functional dependence of the compressive strength of porous materials on the fractal dimension of fracture surfaces. This dependence has also been confirmed experimentally. The explanatory remarks provide a basis for better establishing the model.
\end{abstract}

Keywords: fractal dimension, fracture surfaces, porous materials, compressive strength.

\section{Introduction}

Mandelbrot and his co-workers [1] started fractal research of fracture surfaces of solid materials. After their pioneering work had been published, many other authors $[2,3,4,5]$ tried to correlate the fractal properties of fracture surfaces with the mechanical quantities of materials. Due to the complexity of these surfaces, especially with composite materials, the results of such studies were not consistent, sometimes even contradictory. Good examples of such complicated surfaces are the fracture surfaces of cementitious materials. Nevertheless, they are extensively studied $[6,7]$.

Recently a fractal model of the fracture surfaces of porous materials has been published $[8,9,10]$. Its functionality has been tested and proved with porous cementitious materials. One of the most important results of the model consists in the relation $\sigma=f(D)$ which is the dependence of the compressive strength $\sigma$ of materials on the fractal dimension $D$ of fracture surfaces. This finding may be of practical importance, since it indicates the possibility of estimating compressive strength on the basis of the fractal geometry of fracture surfaces.

The aim of this paper is to provide necessary explanations and comments on particular steps performed within the derivation of the model [8-10] in order to make transparent all its parts. After a short overview of the basic relations of the model (section 2), a series of explanatory sections $3.1-3.3$ follows.

\section{Outline of the model}

A short sketch of the fractal model [8, 9, 10] of fracture surfaces of porous materials is presented here. The content of this section has its source in Ref. [10], which is the most recent presentation of the model.

\subsection{Fractal porosity}

The large class of porous materials possesses at least one common feature, namely, they are composed of grains (particles, globules, etc.) of microscopic size $l$. The grains are usually arranged fractally with number distribution $N(l)$ and fractal dimension $D$

$$
N(l)=\left(\frac{L}{l}\right)^{D}, \quad l<L
$$

Assuming the volume of a globule to be $v=$ const $\cdot l^{3}$ and the volume of a sample $V=$ const $\cdot L^{3}$, the porosity $P$ of the cluster may be derived as follows

$$
\begin{gathered}
P=\frac{V-N \cdot v}{V}=1-N \frac{v}{V}=1-N\left(\frac{l}{L}\right)^{3}= \\
1-\left(\frac{L}{l}\right)^{D}\left(\frac{l}{L}\right)^{3}=1-\left(\frac{l}{L}\right)^{3-D} .
\end{gathered}
$$

In general, the porosity $P$ of a sample with a characteristic size $\Lambda$, stochastically scattered fractal clusters of sizes $\left\{L_{i}\right\}_{i=0,1,2, \ldots, n} \ll \Lambda$ and dimensions $\left\{D_{i}\right\}_{i=0,1,2, \ldots, n}$ reads

$$
P=1-\sum_{i=0}^{n} \xi_{i}\left(\frac{l_{i}}{L_{i}}\right)^{3-D_{i}}, \quad \xi_{i}=m_{i} L^{3} / \Lambda^{3}
$$

where $m_{i}$ is the number of fractal clusters with dimension $D_{i}$.

\subsection{Fractal compressive strength}

The relations for estimating the compressive strength of porous materials published earlier $[11,12]$ rely on porosity $P$ as a main decisive factor. In the fractal model under discussion the Balshin [11] relation $\sigma=\sigma_{o}^{*}(1-P)^{k}$ was used as a starting point. The relation was developed [10] into a more general form

$$
\sigma=\sigma_{o}^{*}\left(1-\frac{P}{P_{c r}}\right)^{k}+s_{o}=\sigma_{o}(1-P-b)^{k}+s_{o},
$$




$$
0 \leq b=1-P_{c r} \leq 1, \quad 0 \leq \sigma_{o}^{*} \leq \sigma_{o}=\frac{\sigma_{o}^{*}}{P_{c r}^{k}}
$$

where $s_{O}$ is the remaining strength which may be caused, among others, by the virtual incompressibility of pore liquids.

Combining (3) and (4), the compressive strength of porous matter appears as a function of the fractal structure

$$
\sigma=\sigma_{o}\left[\sum_{i=0}^{n} \xi_{i}\left(\frac{l_{i}}{L_{i}}\right)^{3-D_{i}}-b\right]^{k}+s_{o}
$$

\subsection{Dimension of fracture surface}

When performing the fracture of a porous material whose inner (volume) structure has fractal dimension $D_{i}$, this structure is projected onto the fracture surface with a lower dimension $D_{i}^{*}<D_{i}$. Provided a fracture surface has its own dimension $S$ and its morphology is 'typical' rather than 'special', the relation between $D_{i}^{*}$ and $D_{i}$ can be expressed [13] as follows

$$
D_{i}=\max \left\{0, D_{i}^{*}+(3-S)\right\}, \quad D_{i}^{*} \leq S<3
$$

where $3-S$ is the co-dimension of the fracture surface. Using (7), the exponent $3-D_{i}$ in Eq. (6) can be replaced by $S-D_{i}^{*}$ and the generalized strength function now reads

$$
\sigma=\sigma_{o}\left[\sum_{i=0}^{n} \xi_{i}\left(\frac{l_{i}}{L_{i}}\right)^{S-D_{i}^{*}}-b\right]^{k}+s_{o}
$$

This function may contain many parameters, so that it is difficult to fit it to the experimental data, because there may be more than one 'reliable' set of parameters $\sigma_{o},\left\{\xi_{i}\right\}_{i=1}^{n}, l_{i}, L_{i}, S, D_{i}^{*}, b, k, s_{o}$. Fortunately, the structure of porous material often contains only one type of grain, i.e. one type of fractal arrangement $(i=1)$ dominates over the solid rest $(i=0)$ which is usually of non-fractal character $\left(D_{o}=3\right)$

$$
\begin{gathered}
\sigma=\sigma_{o}\left[\xi_{1}\left(\frac{l_{1}}{L_{1}}\right)^{S-D_{1}^{*}}+\left(\xi_{o}-b\right)\right]^{k}+s_{o}= \\
\sigma_{o}\left[\xi_{1} \exp \left(\left(S-D_{1}^{*}\right) / A\right)-\gamma\right]^{k}+s_{o}, \\
A=\frac{1}{\ln \left(L_{1} / l_{1}\right)}, \quad \gamma=\left(b-\xi_{o}\right) .
\end{gathered}
$$

\subsection{Experimental tests}

Relation (9) is directly applicable to samples of hydrated Portland cement paste, since it is a composite whose main component (Calcium-Silicate-Hydrate gel) is known for its inner fractal structure. Other components can be assigned to a non-fractal remnant. Therefore, this material was used $[8,9,10]$ to test the functionality of Eq. (9).

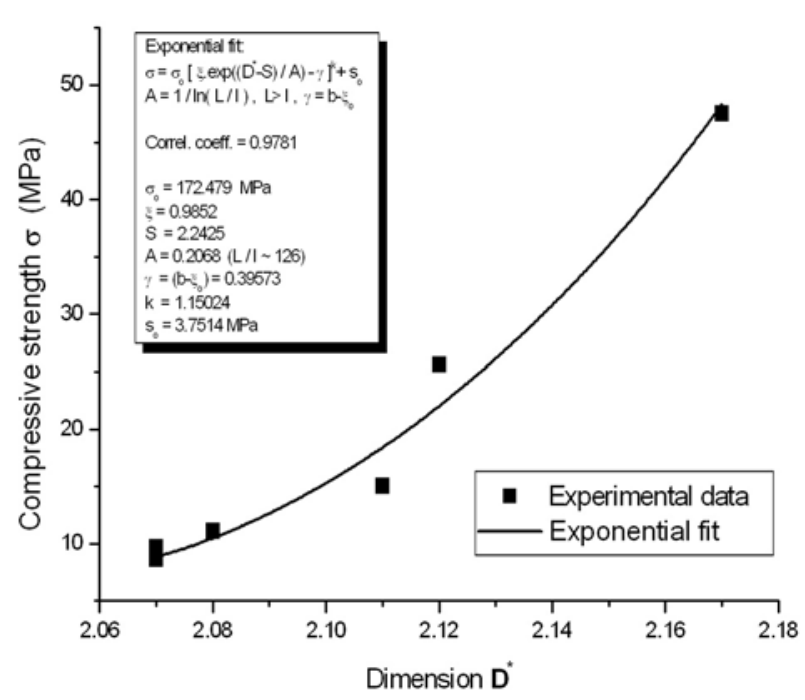

Fig. 1: Dependence of compressive strength on fractal dimension with cement paste [8]

Seventy-two samples of hydrated ordinary Portland cement paste of various water-to-cement ratio $r(0.4,0.6,0.8,1.0,1.2,1.4)$ were prepared. After 28 days of hydration the samples were subjected to three-point bending and were fractured. The fracture surfaces were used for further fractal analysis. The 3-D digital reconstruction of the fracture surfaces was performed using a confocal microscope, and then a series of horizontal sections (contours) were analyzed with resolution $0.2 \mu \mathrm{m}^{2} /$ pixel by means of the standard box-counting method $[8,9,10]$ to obtain a representative $D^{*}$ for the particular surface. The box-counting analyses were performed in the length interval $\langle 2 \mu \mathrm{m}, 300 \mu \mathrm{m}\rangle$. The second parts of the fractured samples were cut into small cubes and subjected to destructive tests to determine their compressive strength values $\sigma$.

It is known that cement pastes of higher waterto-cement ratio suffer from sedimentation of cement clinker grains and bleeding, which may lead to lower homogeneity and modified $\mathrm{w} / \mathrm{c}$ ratio. The influences of these effects on strength measurements have been partly suppressed by the fact that samples with higher $\mathrm{w} / \mathrm{c}$ are localized on the strength curve in the less sensitive region in which the curve is bent and asymptotically approaches the horizontal direction. The surface inhomogeneity has been partly compensated by taking microscopic images from different sites on the surface.

Samples prepared with different water-to-cement ratio $r$ possess different porosity. From cement technology it is well-known that with increasing ratio $r$ the porosity increases. Naturally, this will change the dimensions of the projected patterns $D^{*}$. Six groups of samples with different $r$ means six different $D^{*}$ at which we are able to measure the dependence $\sigma\left(D^{*}\right)$ and check it according to Eq. (9). The result can be seen in Fig. 1 along with all the fitting 
parameters. Since the assumed analytical form (9) of the dependence $\sigma\left(D^{*}\right)$ has been reproduced well, one may conclude that compressive strength is one of those mechanical quantities whose value is 'coded' in the surface arrangement of the fractured samples of porous materials.

\section{Explanatory remarks and discussion}

The following paragraphs provide comments on the proposed concept of fractal compressive strength in order to clarify all its crucial points.

\subsection{Derivation of fractal porosity}

The derivation of fractal porosity starts with Eq. (1), which determines the number $N$ of fractal elements on the length scale $l$. The length $L$ has been taken as a reference scale standing for the largest possible scale on which only one fractal element is present (the so-called initiator, to use Mandelbrot's nomenclature [14]). In short, the object under discussion shows power law behavior (1) only within a limited length interval $(l, L)$ whose borders $l$ and $L$ were coined by Mandelbrot [1] as the 'inner' and 'outer' cutoffs. Beyond this interval the object behaves as an ordinary non-fractal Euclidean body. On the smallest length scale $l$ we can 'see' a great number $(N(l))$ of basic building elements of size $l$, and as we go to larger length scales, the number of corresponding elements decreases. At the length scale $l=L$ there is only one element, i.e. $N_{o}=1$. This is a common property of all self-similar fractals and can be demonstrated very instructively with all deterministic fractals [15], e.g., the Cantor set, Koch curve, Menger sponge, etc.

To explain the origin of Eq. (1), it is necessary to go to the definition of a fractal measure and a fractal dimension. The most general definitions of these quantities are those of Hausdorff [16]. However, his definitions are rather sophisticated and not convenient for computer implementation. For software processing there are some modifications, among which the box-counting procedure is frequently used $[17,18,19,20]$.

The box-counting measure $M$ is given as a sum of $d$-dimensional 'boxes' $\left(l^{d}\right)$ needed to cover the fractal objects embedded in the $E$-dimensional Euclidean space. The boxes are parts of the $d$-dimensional network created in the Euclidean space:

$$
\begin{aligned}
M= & \sum_{i=1}^{N} l^{d}=N \cdot l^{d}=\exp (\ln N) \cdot l^{d}= \\
& {[\exp (\ln l)]^{\frac{\ln N}{\ln l}} \cdot l^{d}=l^{\frac{\ln N}{\ln l}} \cdot l^{d}=l^{d-\frac{\ln N}{\ln (1 / l)}}=}
\end{aligned}
$$

$$
l^{d-D} \rightarrow \lim _{l \rightarrow 0} l^{d-D}=\left\{\begin{array}{cc}
\infty, & d<D \\
0, & d>D
\end{array}\right\} .
$$

The fractal box-counting dimension is defined by the point of discontinuity of the function $M(d)$. According to Eq. (10), this is just the point

$$
d=D=\frac{\ln N}{\ln (1 / l)}
$$

where the measure $M$ abruptly changes its value from infinity to zero. From such a defined dimension $D$ it is easy to express the number $N$ of fractal elements whose size is equal to $l$ or $L$

$$
\begin{aligned}
N(l) & =l^{-D}, \\
N(L) & =L^{-D}=N_{o} .
\end{aligned}
$$

Combining (12) and (13), we obtain

$$
N(l)=N_{o}\left(\frac{L}{l}\right)^{D} .
$$

Bearing in mind that $N_{o}$ belongs to the 'initiator' of the fractal object, i.e. $N_{o}=1$, we obtain

$$
N(l)=\left(\frac{L}{l}\right)^{D}
$$

with a total interval of fractality $(l, L)$. Relation (15) is in fact Eq. (1), which was a starting point in deriving fractal porosity in section 2.1. The functionality of (15) can be easily verified using deterministic fractals [15]. For example, the Koch curve $(D=\ln 4 / \ln 3)$ in its third generation has $N_{3}=4^{3}$ elements with the length $l_{3}=1 / 3^{3}$. The number $N_{3}=$ $4^{3}$ can be obtained from Eq. (15) by inserting $L=1$, $l_{3}=1 / 3^{3}$ and $D=\ln 4 / \ln 3$ which give the following result $N_{3}=\left[1 /\left(1 / 3^{3}\right)\right]^{D}=3^{3 D}=3^{3 \ln 4 / \ln 3}=$ $\mathrm{e}^{3 \ln 3 \cdot \ln 4 / \ln 3}=\mathrm{e}^{3 \ln 4}=4^{3}$ in full agreement with what had been expected. If the length $L$ of the initiator is different from one, however, the result remains unchanged, i.e. $N_{3}=\left[L /\left(L / 3^{3}\right)\right]^{D}=3^{3 D} \ldots$

As far as Eq.(2) is concerned, we may raise a question about its validity if the basic building elements of size $l$ are small spheres tightly packed in the Euclidean space. Due to the compactness of the structure it holds $D=3$. Eq. (2) then yields $P=0$ instead of $P>0$, which would be expected since there are always gaps between spheres, regardless of their type of space arrangement. Here we should bear in mind that spheres of finite diameter cannot generate a true fractal since it requires the presence of an infinitely fine structure, i.e. $l \rightarrow 0$. In such a case Eq. (2) provides $P=1-0^{0}$ which is, however, an uncertain expression that allows no mathematical decision to be made. Nevertheless, the condition $l \rightarrow 0$ ensures that with tight arrangement 
there are no gaps between 'spheres', since 'point-like spheres' completely fill in the Euclidean space and, thus, porosity must be zero. This means that the uncertain expression $P=1-0^{0}$ should also converge to zero. In addition, performing the same procedure with small cubes instead of small spheres, the value $D=3$ (tight arrangement) can be attained even with cubes of finite size $(l>0)$, and the corresponding porosity is then exactly zero $(P=0)$, as required. Therefore, the non-zero porosity in the case of 'tightly' packed spheres of finite size is a consequence of a shape artifact and not of erroneous behavior of Eq. (2).

When dealing with a composite material, not each of its components is a fractal and not each fractal cluster is delocalized over the whole sample. For this reason, it is necessary to assume that the sample consists of sets $(i=0,1,2, \ldots, n)$ of both fractal and non-fractal clusters whose characteristic sizes $L_{i}$ are smaller than or at most equal to the size $\Lambda$ of the sample (Fig. 2). If $m_{i}$ denotes a number of clusters of the $i$-th type (either fractal or non-fractal), the porosity $P$ can be derived by analogy with Eq. (2)

$$
\begin{aligned}
P= & \frac{V-\sum_{i=0}^{n} m_{i} \cdot N_{i} \cdot v_{i}}{V}= \\
& \frac{\Lambda^{3}-\sum_{i=0}^{n} m_{i} \cdot\left(\frac{L_{i}}{l_{i}}\right)^{D_{i}} \cdot l_{i}^{3}}{\Lambda^{3}}= \\
& 1-\sum_{i=0}^{n} \frac{m_{i} \cdot\left(\frac{L_{i}}{l_{i}}\right)^{D_{i}} \cdot l_{i}^{3}}{\Lambda^{3}}= \\
& 1-\sum_{i=0}^{n}\left(\frac{m_{i} L_{i}^{3}}{\Lambda^{3}}\right) \cdot\left(\frac{l_{i}}{L_{i}}\right)^{3-D_{i}}= \\
& 1-\sum_{i=0}^{n} \xi_{i} \cdot\left(\frac{l_{i}}{L_{i}}\right)^{3-D_{i}}
\end{aligned}
$$

where $\xi_{i}=m_{i} L_{i}^{3} / \Lambda^{3}$. Eq. (16) includes all possibilities of fractal, non-fractal or mixed arrangements. For example, when only $n+1$ fractal components exist and are delocalized over the whole sample $\left(L_{i}=\Lambda, m_{i}=1, \xi_{i}=1\right)$, Eq. (16) reads

$$
P=1-\sum_{i=0}^{n}\left(\frac{l_{i}}{L_{i}}\right)^{3-D_{i}}=1-\sum_{i=0}^{n}\left(\frac{l_{i}}{\Lambda}\right)^{3-D_{i}}
$$

If there are only $n+1$ non-fractal compact components localized inside the sample $\left(L_{i}<\Lambda\right)$, then porosity assumes a common 'non-fractal' form

$$
P=1-\sum_{i=0}^{n} \xi_{i}, \quad \xi_{i}=\frac{m_{i} L_{i}^{3}}{\Lambda^{3}}=\frac{v_{i}}{V} .
$$

If fully delocalized compact (i.e., non-fractal) components $\left(L_{i}=\Lambda\right)$ are considered, then, naturally, only one of them can be taken into account since no more than a single fully compact component can fill in the volume of the sample, i.e. $D_{o}=3, L_{o}=\Lambda, m_{o}=1$, $\xi_{o}=1, n=0$

$$
P=1-\xi_{o}=0 \quad \text { (fully compact body). }
$$

Finally, in cases when one fractal component $\left(D_{1}\right)$ and one non-fractal component (compact $D_{o}=3$ ) are present, either of them localized in several sites of the sample $\left(m_{1}-\right.$ fractal clusters, $m_{o}$ - non-fractal clusters), the porosity can be expressed as follows

$$
\begin{aligned}
P & =1-\xi_{1}\left(\frac{l_{1}}{L_{1}}\right)^{3-D_{1}}-\xi_{o}, \\
\xi_{1} & =\frac{m_{1} L_{1}^{3}}{\Lambda^{3}}, \quad \xi_{0}=\frac{m_{o} L_{o}^{3}}{\Lambda^{3}} .
\end{aligned}
$$

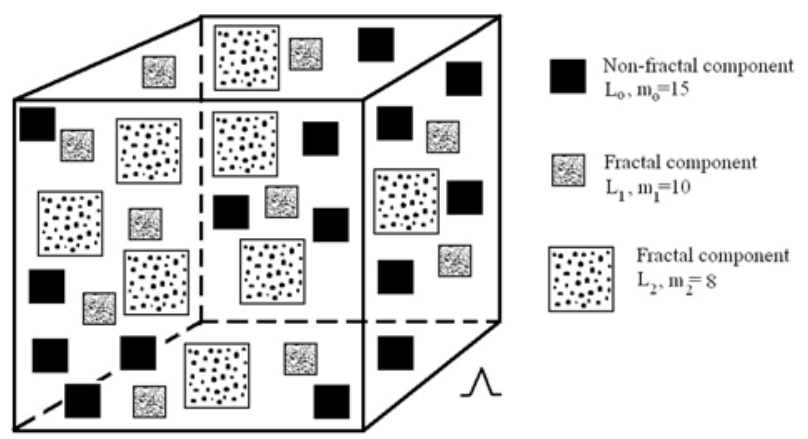

Fig. 2: A scheme of a porous composite material (3 components)

\subsection{Derivation of compressive strength}

In technical literature, many relations have been introduced by various authors. Most of these relations use porosity $P$ as a main governing factor. Let us discuss some relations concerning compressive strength. Balshin [11] suggested a power function $\sigma=\sigma_{o}^{*}(1-P)^{k}$ that was related to porous metallic ceramic materials. His relation is equivalent to the well-known expression of Powers [21]. Ryshkewitch [22] recommended the exponential function $\sigma=\sigma_{o}^{*} \exp (-b P)$, which is in fact an asymptotic form ${ }^{1}(P \rightarrow 0)$ of the Balshin power function. Schiller [23] presented an expression similar to that of Balshin in the form $\sigma=\sigma_{o}^{*}\left[1-\left(P / P_{c r}\right)^{1 / a}\right]$, corrected for a critical porosity $P_{c r}$ at which compressive strength approaches zero. Several other relations are summarized in Ref. [12].

\footnotetext{
${ }^{1}$ The Balshin relation can be rewritten in the Ryshkewitch fomula considering $(1-P)^{k}=\exp [k \cdot \ln (1-P)]$ and restricting to the first term of the Taylor series of the logarithmic function.
} 
There are two important points that should be taken into account when dealing with the compressive strength of porous materials, namely, the so-called critical porosity $P_{c r}$ and partly incompressible pore liquids. Schiller [23] considered the critical porosity $P_{c r}$ as a limiting factor for compressive strength, i.e. $\sigma\left(P_{c r}\right)=0$. But the limit may also be somewhat influenced by the virtual incompressibility of porous liquids. Liquids are displaced in the porous network under the action of an imposed external mechanical load, but narrow pores hinder the liquid movement [24] and due to the virtual incompressibility of the liquid the strength of the structure may be somewhat modified. It is natural that this effect concerns especially quite narrow pores, and with their increasing diameters this effect weakens. However, let us term the modified strength as the remaining strength $s_{o}$. Now it is clear that $P_{c r}$ and $s_{o}$ should be correlated to fulfill the condition $\sigma\left(P_{c r}\right)=s_{o}$. Taking the Balshin relation $\sigma=\sigma_{o}^{*}(1-P)^{k}$ as a good starting point, his form may be generalized by taking into account $P_{c r}$ and $s_{o}$, as follows

$$
\sigma=\sigma_{o}^{*}\left(1-\frac{P}{P_{c r}}\right)^{k}+s_{o}, \quad \sigma\left(P_{c r}\right)=s_{o} .
$$

Now the critical porosity $P_{c r}$ does not represent the absolute limit of strength but it only defines a limit at which the influence of incompressible liquids begins to play a role.

\subsection{Universal exponent of fracture surfaces}

It is important to realize that the fractality of porous materials is determined by their solid skeleton and not by their pores, which are a consequence of the volume arrangement of material components possessing dimensions $\left\{D_{i}\right\}$. As soon as the volume structure is broken and a fracture surface appears, a new topological situation occurs. The volume components $\left\{D_{i}\right\}$ create surface patterns $\left\{D_{i}^{*}\right\}$ with lower dimensions $D_{i}^{*}<D_{i}$. The decrease of the dimensions $\left\{D_{i}\right\}$ can easily be found when the fracture surface is a plane (intersection of the Euclidean plane and the volume fractal component). In this case $D_{i}^{*}=D_{i}-1$, as is well-known. In general, the value of the dimensional shift of a fractal that has originally been embedded in the Euclidean space (E) and then projected onto a subspace $(S<E)$ is called the co-dimension $(E-S)$. When the original space is three-dimensional $(E=3)$ and the subspace two dimensional $(S=2)$, the co-dimension is one $(E-S=1)$, as in the case of intersection of the Euclidean plane with a volume fractal. However, fracture surfaces are not smooth Euclidean planes but rather irregular wavy surfaces. Let us consider the simplest case of fracture of a nonporous fully compact solid, e.g. a pure metal. Such a solid has no fractal volume component, but its frac- ture surface is fractal $\left(2<D_{o}^{*}<3\right)$, as has been shown elsewhere [25]. On the other hand, when a solid consisting of one delocalized fractal component $(D)$ is broken, the dimension $D^{*}$ of the corresponding fracture surface is equal to the dimension $D^{*}$ of the fractal projection onto an 'imaginary' subspace $S$, i.e. $D^{*}=D-(3-S)$. The dimension $S$ of the subspace can be calculated from the dimensions of the volume fractal $D$ and its surface projection $D^{*}$, i.e. $S=3-\left(D-D^{*}\right)$, provided there are techniques for determining $D$ and $D^{*}$. Similarly, if a solid is composed of more than one fractal component $\left\{D_{i}\right\}$, the dimensions of their surface projections $\left\{D_{i}^{*}\right\}$ are given as follows

$$
D_{i}^{*}=D_{i}-(3-S) \Rightarrow 3-D_{i}=S-D_{i}^{*}
$$

This relation has been used when going from Eq. (6) to Eq. (8), i.e. from volume fractals to their surface projections. The dimensions of surface projections $D_{i}^{*}$ are 'measurable' e.g. using the confocal technique, if these components are extended over different length scales and do not overlap each other. In our case of hydrated cement paste there is one fractal component (Calcium-Silicate-Hydrate gel) that dominates over the other non-fractal components, and this simplifies the computations according to Eq. (9).

There is no reason why the mentioned subspace has to be of the Euclidean type. It can also be of fractal type, i.e. its dimension $S$ can be not only an integer but also a non-integer number. And this is the case of compact metals possessing the dimension $D_{i}=3$, for which Eq. (22) gives $D_{i}^{*}=S$. Bouchaud, Lapasset and Planès [25], when investigated metallic fractures, found $D_{i}^{*}=2.2$ which means $S=2.2$. In our case of porous Calcium-Silicate-Hydrates the dimension $S$ has also been found in the same rank $S \approx 2.2$, although these two materials are quite different. The idea that $S$ may be a universal exponent related to fracture surfaces has been indicated previously [25], and our results seem to support it, nevertheless, this concept should be studied further. If future experiments confirm the concept, then it will be necessary to distinguish carefully between the two types of exponents $S$ and $D_{i}^{*}$. The former exponent $S$ is a relatively stable and probably universal exponent which would be directly measurable if the sample were fully compact (non-porous and non-fractal), i.e. a perfect Euclidean body. The dimension $S$ seems to depend more on the fracture process itself than on structural components. The latter exponents are the dimensions $\left\{D_{i}^{*}\right\}$ of surface projections. They vary with the properties of materials, which has been illustrated in the previous studies $[9,10]$ by using a series of samples of different compressive strength. These studies have simultaneously confirmed that fracture surfaces bear information on the compressive strength of porous materials (Fig. 2). 


\section{Conclusion}

The fractal model of compressive strength may be applicable to all fractal porous materials. If the particular material is composed of a single fractal component, the model contains only a few parameters that can be easily fitted to experimental data. However, when more fractal components are present, many parameters have to be fitted and there may arise numerical problems in selecting their 'right' values among all the options, each of which satisfies the optimalizing criteria equally well. There is no general numerical procedure that will guarantee such a right selection of values. In these cases an intuitive and heuristic approach, supported by physical reasoning, may be instrumental in finding an optimum solution.

\section{Acknowledgement}

This work was supported by Grant no. ME09046 provided by the Ministry of Education, Youth and Sports of the Czech Republic.

\section{References}

[1] Mandelbrot, B. B., Passoja, D. E., Panllay, A. J.: Nature, 1984, vol. 308, p. 721.

[2] Balankin, A. S. et al.: Phys. Rev., 2005, E 72, 065101R.

[3] Marconi, V. I., Jaga, E. A.: Phys. Rev., 2005, E $\mathbf{7 1}, 036110$.

[4] Bohn, S. et al.: Phys. Rev., 2005, E 71, 046214.

[5] Bouchbinder, E., Kessler, D., Procaccia, I.: Phys. Rev., 2004, E 70, 0461107.

[6] Yan, A., Wu, K.-R., Zhang, D., Yao, W:. Cem. Concr. Res., 2003, vol. 25, p. 153.

[7] Wang, Y., Diamond, S.: Cem. Concr. Res., 2001, vol. 31, p. 1385.

[8] Ficker, T.: Acta Polytechnica, 2007, vol. 47, p. 27.

[9] Ficker, T. Europhys. Lett., 2007, vol. 80, $16002-$ p1.

[10] Ficker, T.: Theoret. Appl. Fract. Mech., 2008, vol. 50, p. 167.
[11] Balshin, M. Y.: Dokl. Akad. Nauk SSSR, 1949, vol. 67, p. 831 (in Russian).

[12] Narayanan, N., Ramamurthy, K.: Cem. Concr. Res., 2000, vol. 22, p. 321.

[13] Falconer, K. J.: Professor of Mathematics, University of St. Andrews, Scotland - private communication.

[14] Mandelbrot, B. B.: Fractal Geometry of Nature. San Francisco, Freeman, 1982.

[15] Ficker, T., Benešovský, P.: Eur. J. Phys., 2002, vol. 23, p. 403.

[16] Hausdorff, F.: Math. Ann., 1919, vol. 79, p. 157.

[17] Ficker, T., Druckmüller, M., Martišek, D.: Czech. J. Phys., 1999, vol. 49, p. 1445.

[18] Ficker, T.: J. Phys. D: Appl. Phys., 1999, vol. 32, p. 219.

[19] Ficker, T.: IEEE Trans. Diel. El. Insul., 2003, vol. 10, p. 700 .

[20] Ficker, T.: J. Phys. D: Appl. Phys., 2005, vol. 38, p. 483 .

[21] Ficker, T., Němec, P.: Porosity and Strength of Cement Materials. In Proceedings of the International Workshop on Physical and Material Engineering 2006, Bratislava, Slovak University of Technology, 2006, p. 30-33.

[22] Ryshkewitch, E.: J. Am. Ceram. Soc., 1953, vol. 36, p. 65.

[23] Schiller, K. K.: In: Walton, W. H. (Ed.) Mechanical Properties of Non-Metallic Brittle Materials. London, Butterworths, 1958, pp. 35-45.

[24] Scherer, G. W.: Cem. Concr. Res., 1999, vol. 29, p. 1149.

[25] Bouchaud, E., Lapasset, G., Planès, J.: Europhys. Lett., 1990, vol. 13, p. 73.

Prof. RNDr. Tomáš Ficker, DrSc.

Phone: +420 541147661

E-mail: ficker.t@fce.vutbr.cz

Department of Physics

Faculty of Civil Engineering

University of Technology

Žižkova 17, 66237 Brno, Czech Republic 